FINAL REPORT:

\title{
Project: Non-Invasive Treatment of Pediatric Neurological Disorders using MR-guided Focused Ultrasound (MRgFUS) - Part 1 Pediatric Skull Characterization and Re-Focusing
}

\section{Introduction:}

Pediatric neurological diseases such as stroke and epilepsy are disproportionally common: stroke is highest in the perinatal period and occurs in 1 in 3000 live births. Epilepsy affects $1 \%$ of all children and up to $30 \%$ of these will develop drug resistant seizures leading to a lifetime of medical disability. Neonatal and pediatric skulls have unique anatomical characteristics such as an open fontanelle, a thinner skull and a reduced acoustic attenuation. The project hypothesizes that these characteristics make them ideal candidates for MRgFUS treatment, since their natural features simplifies the challenge of transcranial sonication and reduces the need for large hemispherical transducers.

As there is minimal literature on the subject, the first part of the project was to perform the acoustic characterization of pediatric skulls ranging from 0 to 16 years old. Then the aim was to study the acoustic propagation near these skulls and develop an algorithm for refocusing across these skulls. In this report we present our current results regarding insertion loss (IL) and time of flight (TOF) delay with two skull specimens. The speed of sound is also calculated for the two skulls. A phantom skull is also characterized and compared to the corresponding cadaveric skull. With the acoustic propagation through the skulls and the refocusing algorithm still being a work in progress, we present here our first observations. Finally we describe our first in vivo study on the piglet with a setup mimicking the neonate skull for the treatment of intraventricular hemorrhage (IVH).

\section{Pediatric skull library:}

Two cadaveric pediatric skulls have been obtained: A neonate skull with an open fontanelle and an 8year old skull (Figure 1). Two phantom skulls, made from a bone-mimicking ceramic, representing the 8year old skull and the neonate skull have also been manufactured by the group of Dr. Roman Maev from the University of Windsor [1].
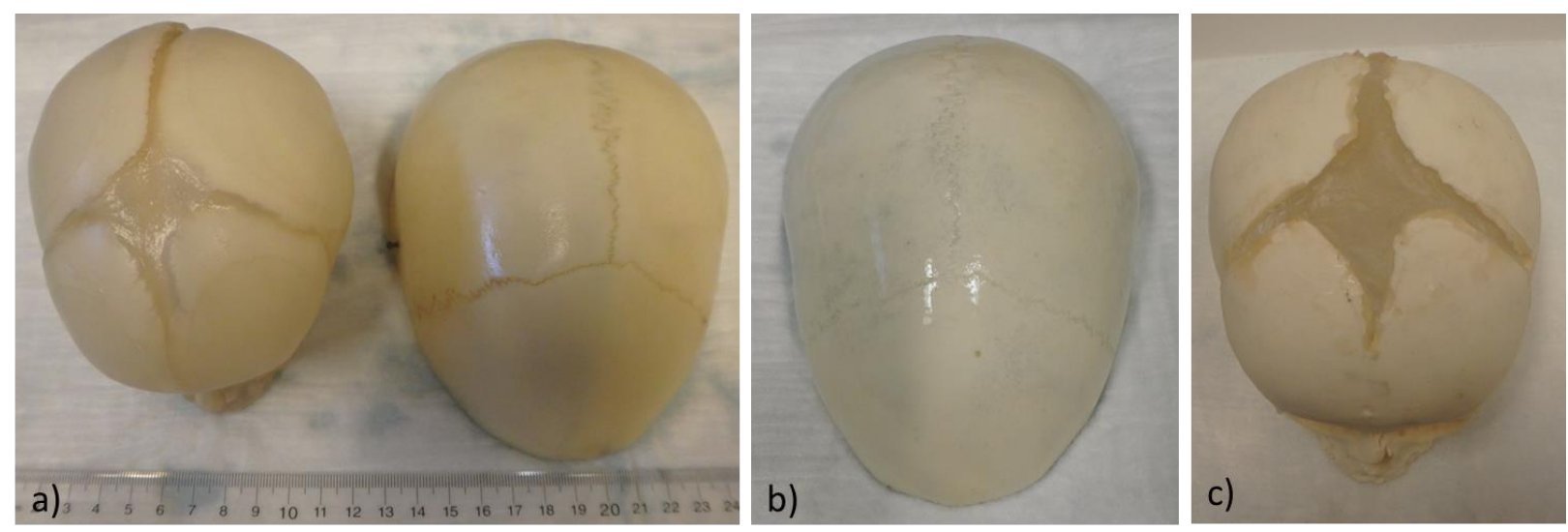

Figure 1: Pediatric skull library: a) on the left the neonate skull, on the right the 8-year old skull, b) the 8-year old phantom skull, c) the neonate phantom skull 


\section{Pediatric skull characterization methods:}

A $0.2 \mathrm{~mm}$ needle acoustic hydrophone is placed in a tank of degassed water and aligned to the geometric focus of a clinical HIFU transducer (Philips Sonalleve V1). The signals of the 256 elements of the phased array transducer are acquired as a baseline measurement using this hydrophone and the MatHIFU software toolkit [2]. The degassed skull is placed inside the tank between the hydrophone and the transducer. New acquisitions are performed for different angular orientations of the skull according to the sagittal and coronal axes in the range of $\pm 15^{\circ}$ (Figure 2). Insertion losses (IL) and time-of-flight (TOF) delays due to the skull and the fontanelle, if present, are deducted from these measurements performed at $1 \mathrm{MHz}, 1.2 \mathrm{MHz}$ and $1.45 \mathrm{MHz}$. Time-of-flight (TOF) delay due to the skull was calculated by the difference between the skull and baseline TOF measurements for each orientation and each frequency. The insertion loss (IL) is obtained by calculating the difference between the root mean square (RMS) amplitude of the skull signals and the baseline RMS amplitude for each orientation and each frequency.

MRI acquisitions of the skull and the transducer positioning were performed for each skull orientation using a large dual-channel flex coil in a Philips Achieva 3.0T TX scanner. A segmented CT skull was then rigidly co-registered with the MRI skull to obtain a better spatial resolution. TOF delay and IL values obtained for each transducer element were then projected onto the CT skull surface. The registration of the CT skull over the MRI skull gives access to the thickness of the bones crossed by the ultrasound beam. The speed of sound is deducted from the measured thickness and TOF delays.

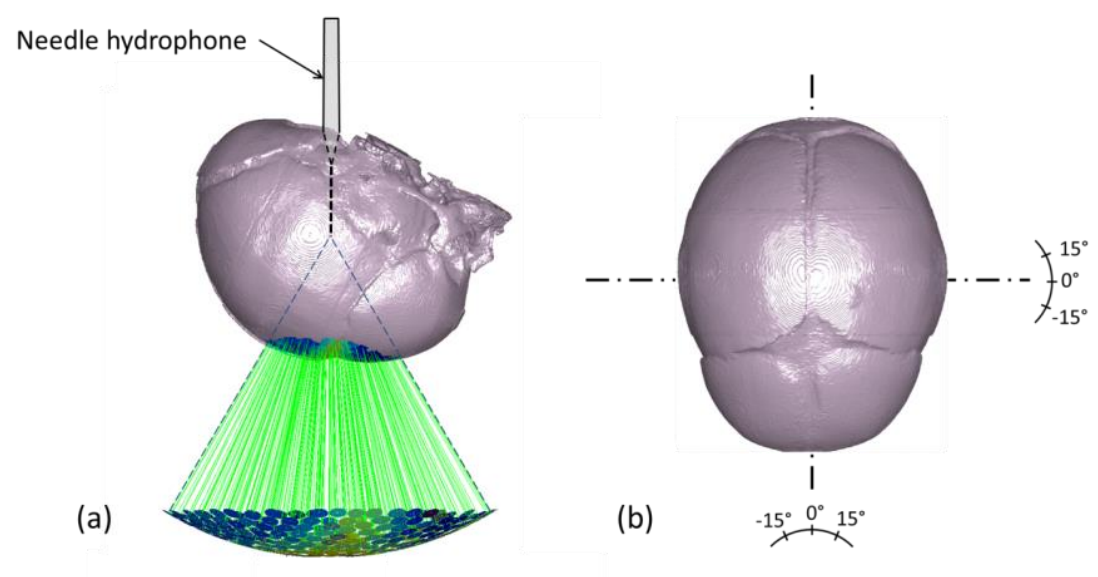

Figure 2: (a) Illustration of the experimental set up showing the skull positioning at $0^{\circ}$ between the hydrophone and the transducer. The green lines represent the beam paths crossing the skull surface; (b) Illustration of the different angular orientations of the skull. 


\section{A. Acoustic properties of the neonate skull:}

\section{Time-of-flight (TOF) delays:}

The fontanelle can be easily discriminated from the bone areas as the TOF delay in that region is very low (TOF delay $y_{\text {fontanelle }}=-0.08 \pm 0.02 \mu$ s over the frequency range) (Figure 3 ).

The TOF delay is constant over the frequency range and is globally the same for all bone areas (TOF delay frontal $=-0.16 \pm 0.02 \mu \mathrm{s}$ and TOF delay parietal $=-0.15 \pm 0.02 \mu \mathrm{s}$ ) (Figure 4).

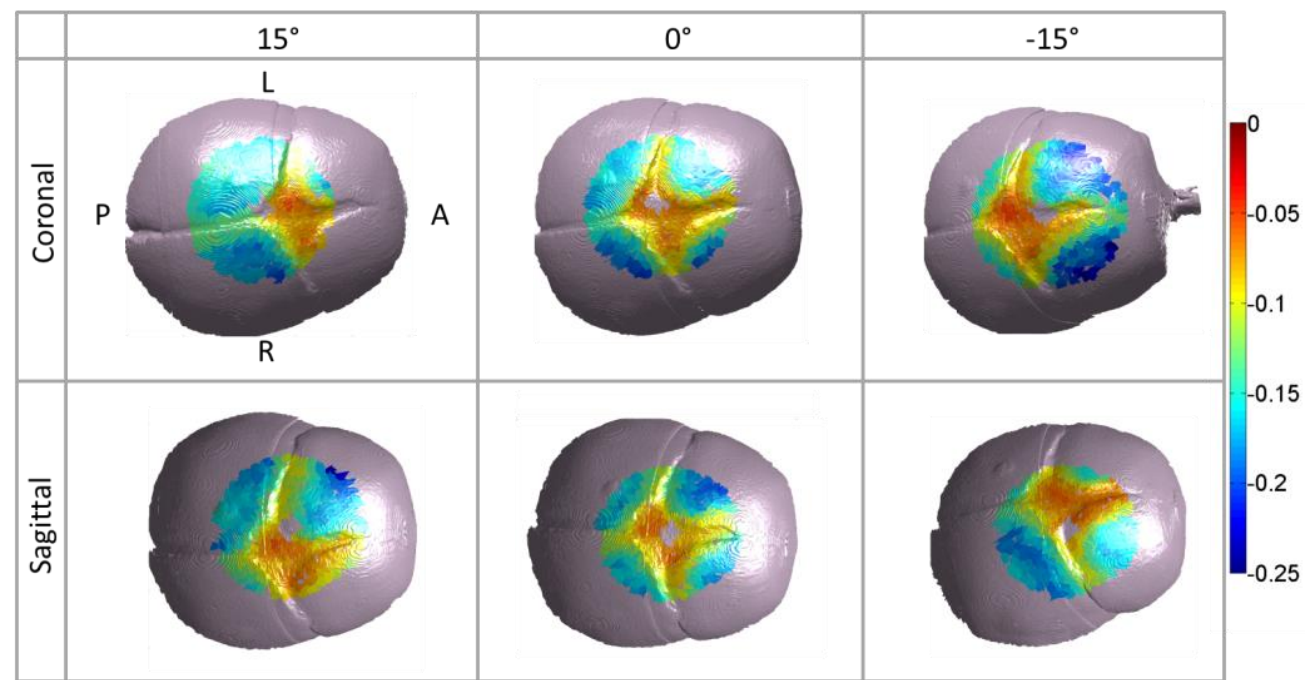

Figure 3: Average TOF delay values ( $\mu \mathrm{s})$ obtained at 1.2 $\mathrm{MHz}$ for each transducer element for each skull orientation.

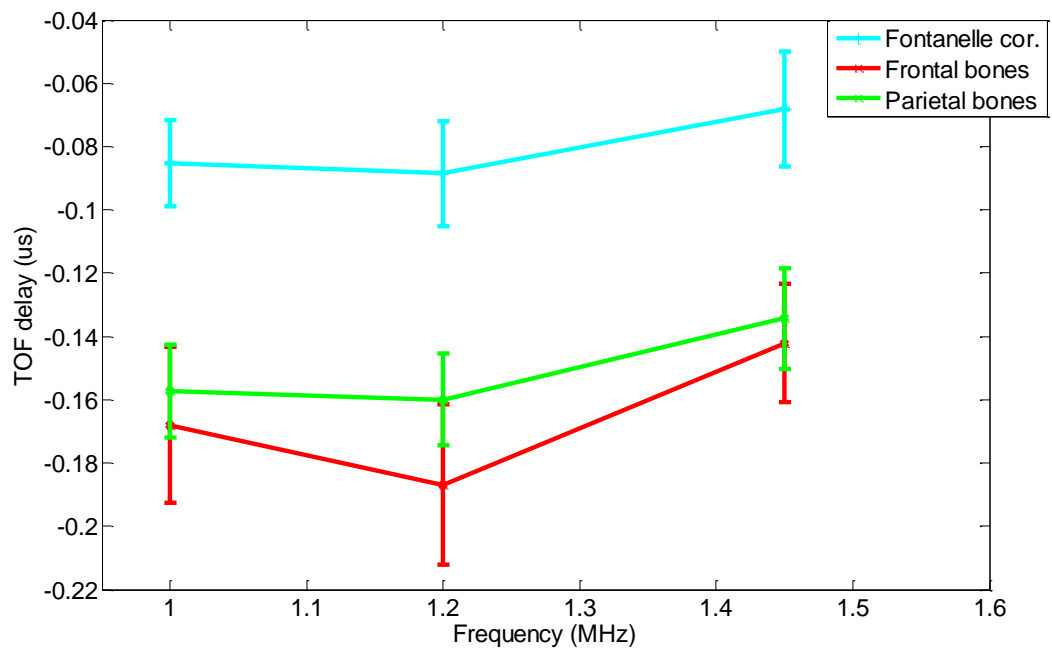

Figure 4: Evolution of the TOF delay ( $\mu \mathrm{s})$ with the frequency $(\mathrm{MHz})$ for the different areas of interest. The average value and its standard deviation are represented for each area. 


\section{Insertion loss (IL):}

Higher variability of the IL values compared to the TOF delay values is observed (Figure 5). The fontanelle is still well demarcated as the IL in this area is very low (ILfontanelle $=0.8 \pm 0.9 \mathrm{~dB}$ over the frequency range).

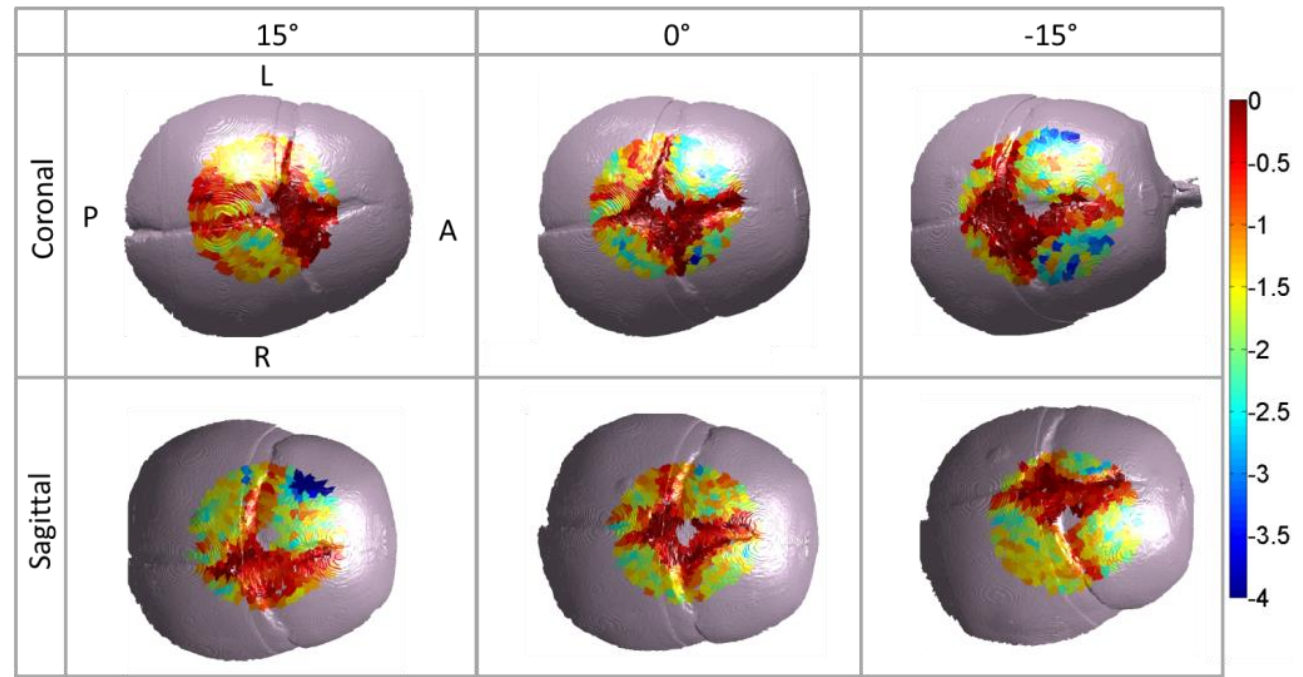

Figure 5: Average IL values ( $\mu \mathrm{s})$ obtained at $1.2 \mathrm{MHz}$ for each transducer element for each skull orientation.

Frontal bones lead globally to higher IL than parietal bones $\left(\|_{\text {frontal }}=2.7 \pm 1 \mathrm{~dB}\right.$ against $\|_{\text {parietal }}=1.9 \pm 0.7$ $\mathrm{dB}$ over the frequency range). The maximum $\mathrm{IL}$ value found is $3 \pm 1 \mathrm{~dB}$ at $1 \mathrm{MHz}$ and $1.45 \mathrm{MHz}$ in the frontal bones (Figure 6). The IL decreases at 1.2 MHz for the frontal and parietal bones. This tendency is confirmed by looking at the values of some random points in these areas.

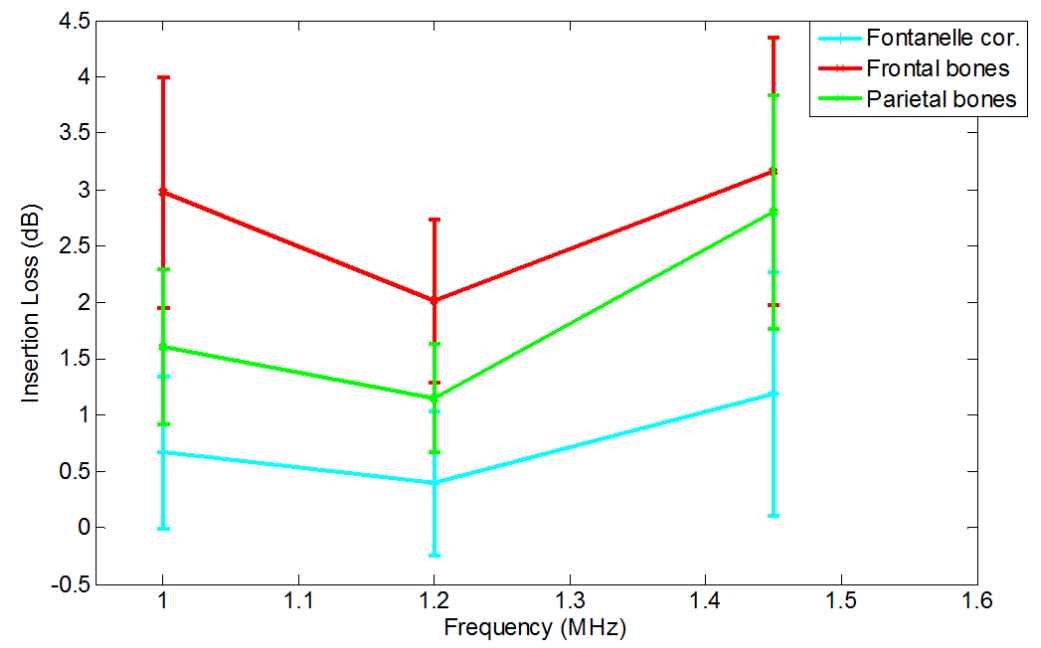

Figure 6: Evolution of the IL ( $\mathrm{dB})$ with the frequency $(\mathrm{MHz})$ for different areas of interest. The average value and its standard deviation are represented for each area. 


\section{Speed of sound:}

For the frontal bones, the average speed of sound at $1.2 \mathrm{MHz}$ is $2025 \pm 136 \mathrm{~m} \cdot \mathrm{s}^{-1}$. For the parietal bones, the average speed of sound at $1.2 \mathrm{MHz}$ is $2041 \pm 154 \mathrm{~m} \cdot \mathrm{s}^{-1}$. These results are in good agreement with the literature $\left(2010<c_{\text {bone }}<2060 \mathrm{~m} \cdot \mathrm{s}^{-1}\right.$ for $0.3-0.8 \mathrm{MHz}$ frequency range [3]).

\section{Discussion:}

Aberration and attenuation effects due to the fontanelle are very low ( $\mathrm{LL}_{\text {fontanelle }}=0.8 \pm 0.9 \mathrm{~dB}$ and TOF delay $_{\text {fontanelle }}=-0.08 \pm 0.02 \mu$ s over the frequency range). IL, TOF delays values and the speed of sound of the neonate skull are low compared to adult skull values found in the literature $\left(25 \mathrm{~dB}\right.$ at $1 \mathrm{MHz}<\|_{\text {adult skull }}$ $<42 \mathrm{~dB}$ at $1.45 \mathrm{MHz}[3]$ and TOF delayadult skull $\approx-1.7 \mu \mathrm{s}$ at $1.4 \mathrm{MHz}$ [4] and $\mathrm{c}_{\text {cortical }}=2504 \pm 120 \mathrm{~m}^{-\mathrm{s}^{-1}}$ at 1.4 $\mathrm{MHz}[4])$. The neonate skull constitutes a weak acoustic barrier and its fontanelle can even act as an acoustic window.

\section{B. Acoustic characterization of the 8-year old skull: \\ 1. TOF delays:}

The TOF delay is constant over the frequency range and is globally the same for all bone areas (TOF delay $_{\text {parietal }}=-0.70 \pm 0.08 \mu \mathrm{s}$ ). The TOF delay is slightly higher for the sagittal suture (TOF delay suture $=-$ $0.74 \pm 0.28 \mu \mathrm{s}$ ) (Figure 7). If the TOF delay is homogeneous in the bone areas and high variability of the TOF delay values was noted in the sagittal suture area (Figure 8). This can be due to the internal structure of the suture which is still a bone growth site at this age and is not completely fused. It can also be due to bubbles being trapped even after several days of degassing.

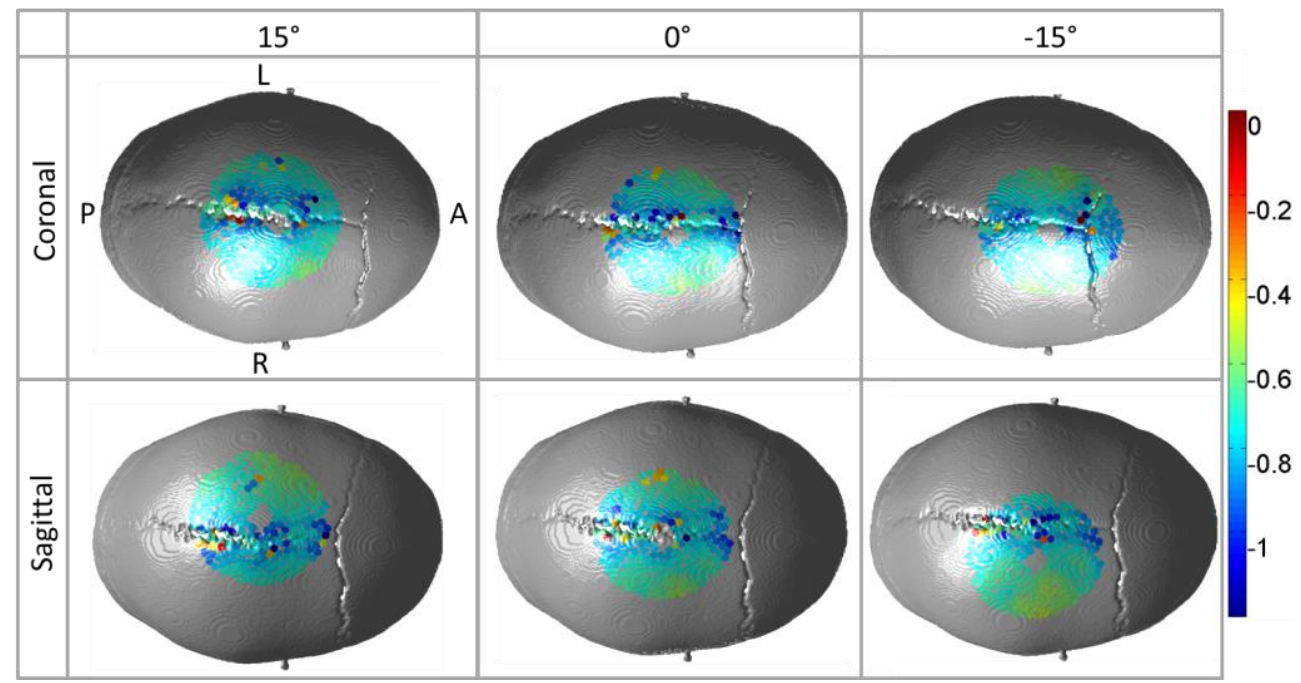

Figure 7: Average TOF delay values ( $\mu$ s) obtained at 1.2 MHz for each transducer element for each skull orientation. 


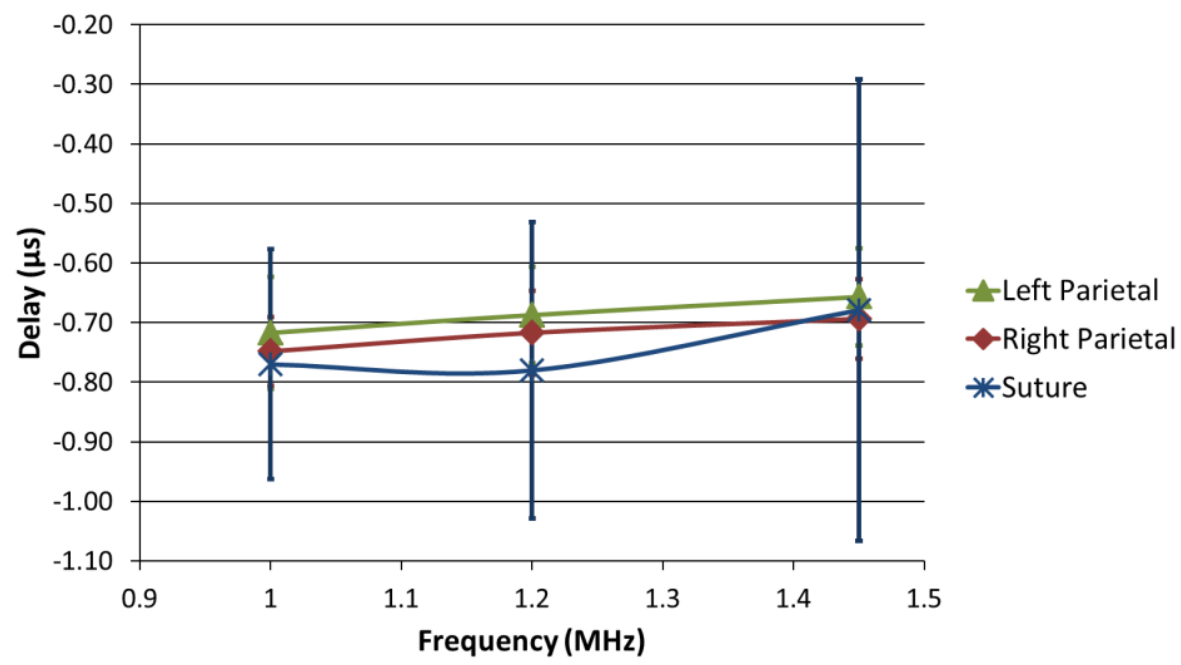

Figure 8: Evolution of the TOF delay ( $\mu \mathrm{s})$ with the frequency $(\mathrm{MHz})$ for the different areas of interest. The average value and its standard deviation are represented for each area.

\section{Insertion loss:}

The average IL is the same for all bone areas and increases with the frequency from $7 \pm 1.3 \mathrm{~dB}$ at $1 \mathrm{MHz}$ to $10.3 \pm 1.5 \mathrm{~dB}$ at $1.45 \mathrm{MHz}$. However the IL values are heterogeneous over the bone surface (Figure 9). Furthermore, the sagittal suture leads to high and heterogeneous IL values increasing also with the frequency from $10.7 \pm 1.5 \mathrm{~dB}$ at $1 \mathrm{MHz}$ to $16.7 \pm 2.2 \mathrm{~dB}$ at $1.45 \mathrm{MHz}$ (Figure 10).

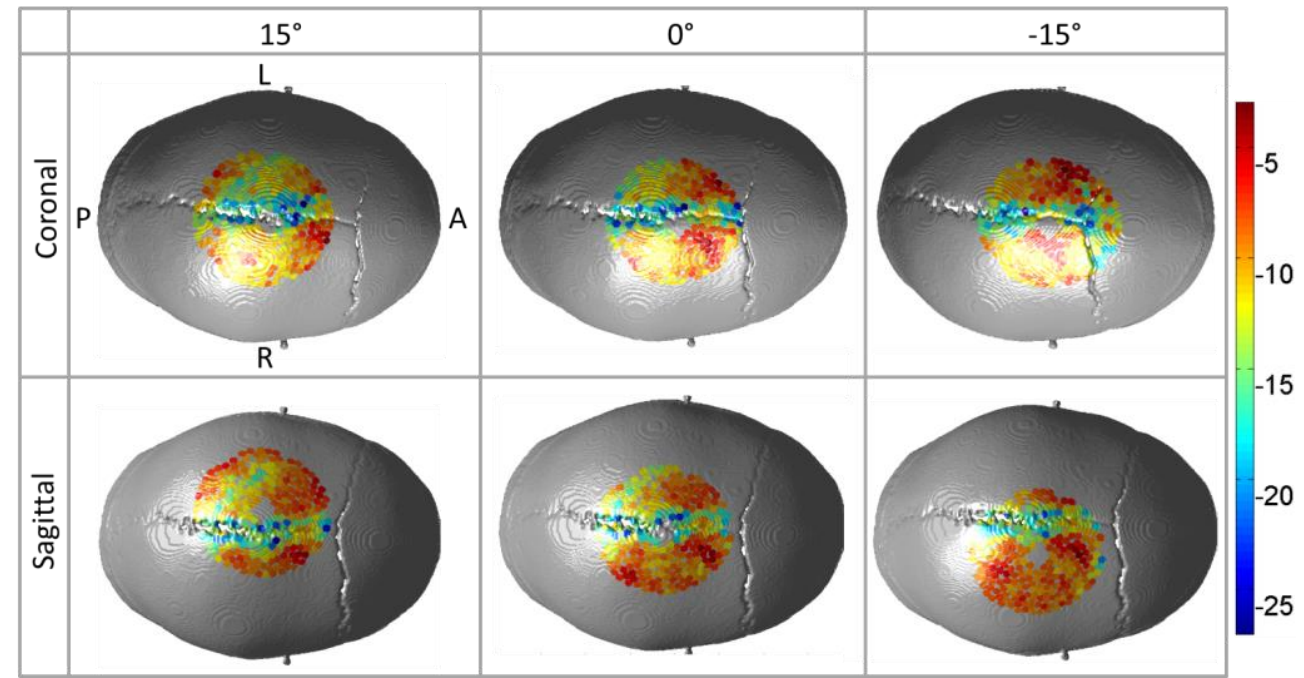

Figure 9: Average IL values (us) obtained at 1.2 MHz for each transducer element for each skull orientation. 


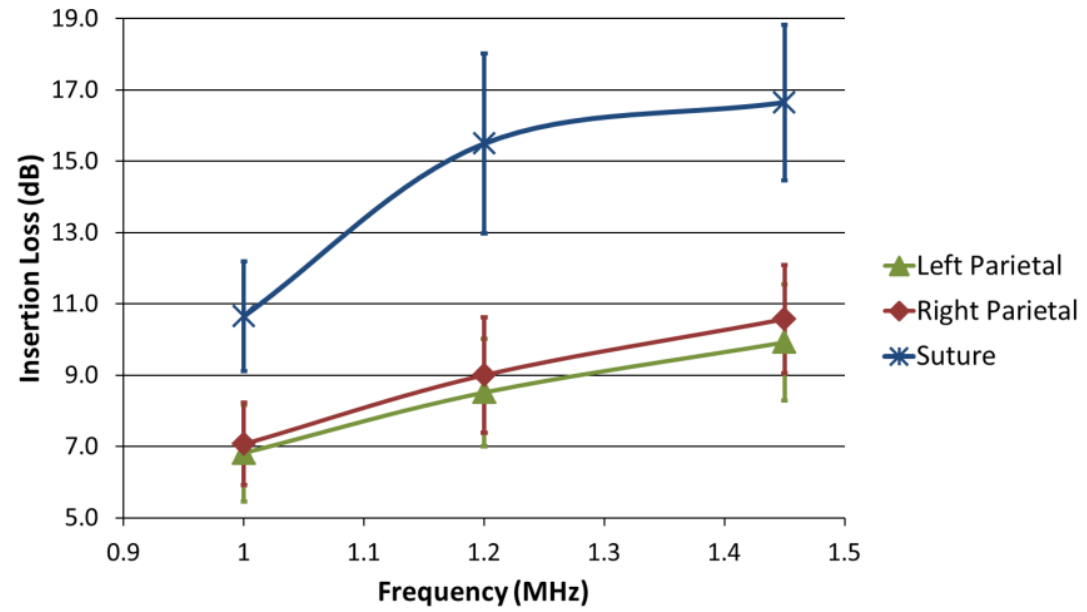

Figure 10: Evolution of the IL ( $\mathrm{dB})$ with the frequency $(\mathrm{MHz})$ for different areas of interest. The average value and its standard deviation are represented for each area.

\section{Speed of sound:}

For the left parietal bones, the average speed of sound at $1 \mathrm{MHz}$ is $2253 \pm 189 \mathrm{~m} . \mathrm{s}^{-1}$. For the right parietal bones, the average speed of sound at $1 \mathrm{MHz}$ is $2311 \pm 226 \mathrm{~m} \cdot \mathrm{s}^{-1}$

\section{Discussion:}

As expected, the 8-y.o. skull bones have higher IL and TOF delays than the neonate ones but are still low compared to the adults ones found in the literature ([3], [4]). The speed of sound globally matches the prediction models based on the density [5]. The structure of the sagittal suture seems to have an impact on the propagation of ultrasound. This anatomical element has to be taken into account in future numerical work. 


\section{Acoustic characterization of the 8-year old phantom skull: \\ 1. Comparison of the TOF delay and IL values with the real skull values}

The average TOF delay of the phantom is $-1.1 \pm 0.3 \mu$ s at $1.2 \mathrm{MHz}$ versus $-0.70 \pm 0.08 \mu$ s for the real skull bone areas, resulting in a $57 \%$ increase of the delay. However the TOF delay of a small area on the right parietal bone near the coronal suture seems to match well with the homogeneous TOF delay of the real skull (Figure 11).
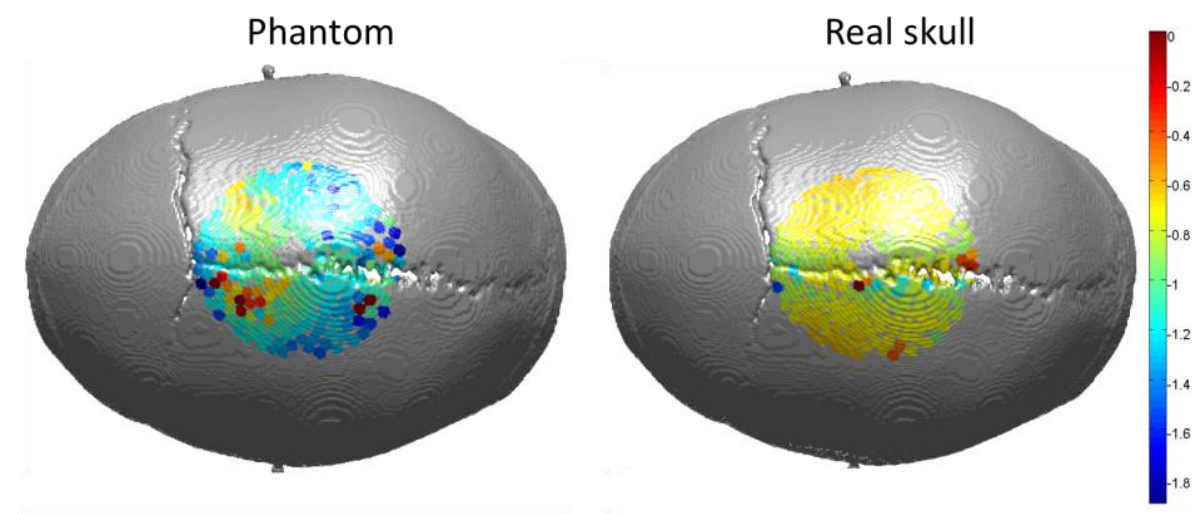

Figure 11: Comparison of the TOF delay ( $\mu \mathrm{s}$ ) of the phantom and the real skull at $1.2 \mathrm{MHz}$

The average IL of the phantom is $14.1 \pm 2.6 \mathrm{~dB}$ at $1.2 \mathrm{MHz}$ versus $9 \mathrm{~dB}$ for the real skull bone areas. But here again, the IL delay of a small area on the right parietal bone near the coronal suture seems to match well with the average IL of the real skull (Figure 12).
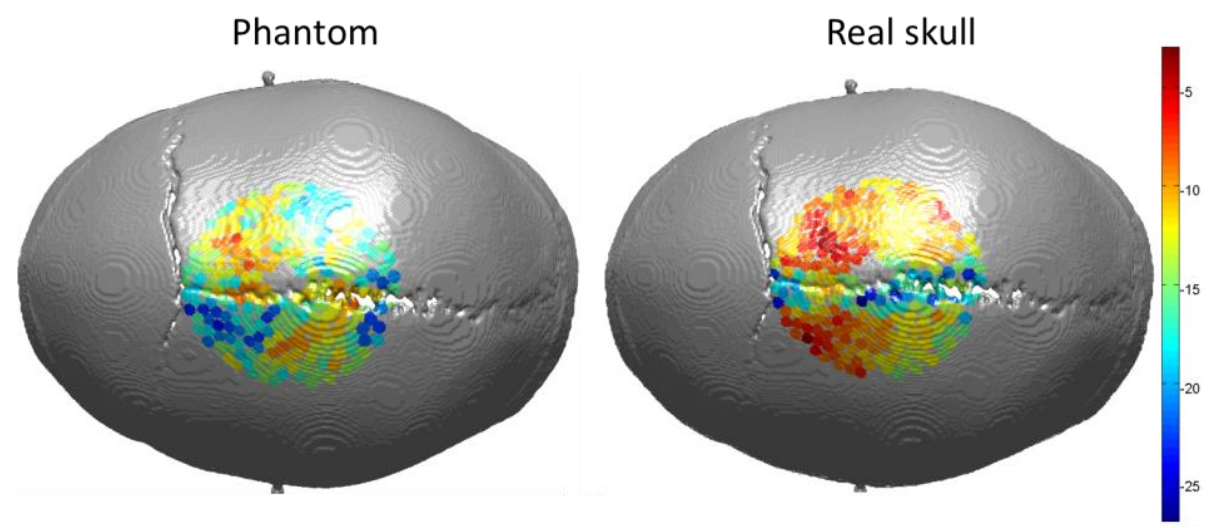

Figure 12: Comparison of the IL (dB) of the phantom and the real skull at $1.2 \mathrm{MHz}$

\section{Discussion}

Based on the CT scan of the real skull, this phantom was manufactured using bone-mimicking material, [1] which was developed based on adult bone samples. The structure of this 8-year old skull is different than the one of an adult skull. It is not yet a completely differentiated flat bone structure (corticaltrabecular-cortical) found in adults but a mix of mostly cortical bone that transitions to a full flat bone structure [6]. These results suggest good matching in mostly cortical bone areas. A CT scan of the phantom will have to be performed to compare the thickness and composition of the two skulls in order to improve the composition of the phantom. 
A new phantom based on the CT scan of the neonate skull has been manufactured (Figure 1c). This phantom is only made of cortical bone-mimicking material and should be close to the real neonate skull based on these results. Testing on the neonatal skull phantom are ongoing.

\section{Refocusing in pediatric skulls}

\section{A. Numerical modeling}

\section{Neonate skull}

Pressure fields at the focal spot were simulated with and without introducing TOF delay and IL created by the neonate skull at $1.2 \mathrm{MHz}$. Insertion of the skull in the beam path leads to a $-0.8 \mathrm{~mm}$ shift of the focal spot along the acoustic axis and to an amplitude loss of $31.8 \%$ or $3.3 \mathrm{~dB}$. After amplitude compensation based on the mean amplitude (1.15X multiplier) and without refocusing, the amplitude loss is only $9.3 \%$ or $0.8 \mathrm{~dB}$ and the focal spot is still shifted of $-0.8 \mathrm{~mm}$ along the acoustic axis.

The aberrations created by the neonate skull are very small and the need for a refocusing algorithm when treating a neonate could be discussed. This is particularly true for treatments performed using ultrasound pulses: IVH treatment for example. The skin temperature increase should be simulated if thermal ablation is considered.

\section{8-year old skull}

Pressure fields at the focal spot were simulated with and without introducing TOF delay and IL created by the 8-year old skull at $1 \mathrm{MHz}$. Insertion of the skull in the beam path leads to a $1.2 \mathrm{~mm}$ shift of the focal spot along the acoustic axis and to an amplitude loss of $81.8 \%$ or $14.8 \mathrm{~dB}$. After amplitude compensation based on the mean amplitude (2.1X multiplier) and without refocusing, the amplitude loss is $19.1 \%$ or $1.84 \mathrm{~dB}$ and the focal spot is still shifted of $1.2 \mathrm{~mm}$ along the acoustic axis. When the elements facing the sagittal suture are turned off and a compensation of the amplitude is applied based on the mean amplitude of the remaining elements (2.5X multiplier), the amplitude loss is $16.2 \%$ or 1.54 $\mathrm{dB}$ and the focal spot is shifted of $1.4 \mathrm{~mm}$ along the acoustic axis.

Based on the previous results (III.B.2), it is essential to avoid the sagittal suture to prevent any skin damage. However the amplitude compensation factor to apply is relatively high. A refocusing algorithm seems necessary for this kind of skull.

\section{B. In vivo experiments on piglets}

A total of 4 IVH piglet models were completed based on previous testing where IVH clots were developed in both the right and left ventricles ([7], [8]). For the models, the amount of blood injected was based on the weight of the piglet $(1.25 \mathrm{cc} / \mathrm{kg})$ where total infusion amount ranged from $5.75 \mathrm{cc}$ to 7.5cc. T1-weighted, T2-weighted and T2*-weighted images confirmed the location and placement of the clots (Figure 13). Average clot volume $=0.718 c c$ (derived from imaging). 


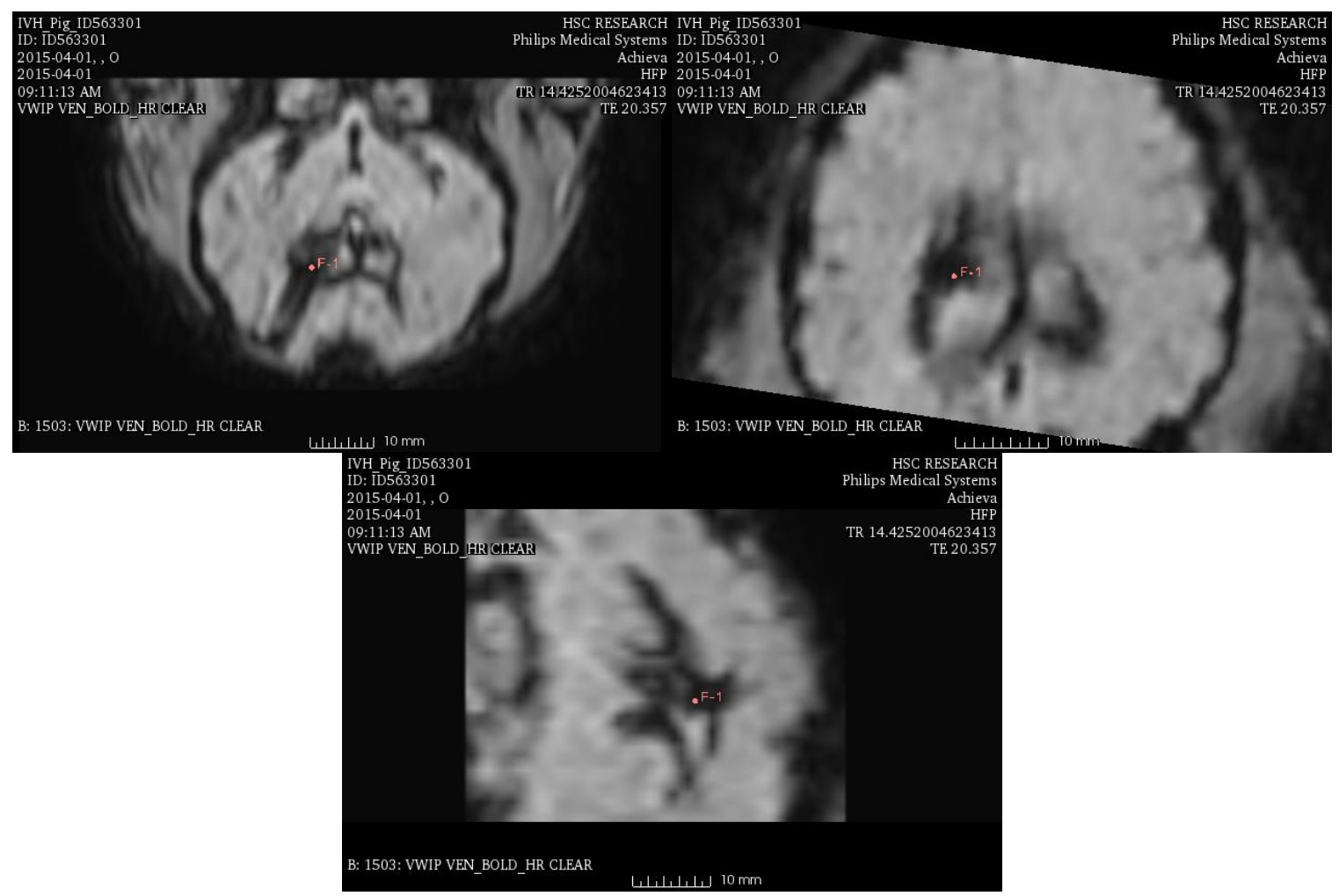

Figure 13 - Top Left: T2*-weighted Coronal, Top Right: T2*-weighted Axial, Bottom: T2*-weighted Sagittal

In preparation for MRgFUS treatment, the thickness of the piglet skull was compared to a cadaveric neonatal skull sample where it was noted that the piglet skull was much thicker $(2-3 \mathrm{~mm}$ vs $<1 \mathrm{~mm})$. Prior neonatal skull characterization had shown there was minimal impact to the ultrasound transmission. Thus to replicate the same treatment scenario, a craniotomy was performed on the piglets to recreate the skull opening (fontanelle) while also mimicking the thin neonate skull. After craniotomy, piglets were placed supine feet first on the MR table with surface coils for MRgFUS imaging on the Philips Sonalleve system.

$\mathrm{T} 2-\mathrm{w}$ and $\mathrm{T} 2 *-\mathrm{w}$ imaging were used to localize the clot and $2 \mathrm{~mm}$ treatment cells were placed throughout the clot region. The acoustic parameters used for the treatment ranged from $750 \mathrm{~W}$ to 850 $\mathrm{W}$ of acoustic power, $0.8 \%$ duty cycle and pulse repetition of $28 \mathrm{~ms}$ at $1.2 \mathrm{MHz}$. Post-treatment T2-W and $2^{*}$-w showed significant areas of hyper-intensity (Figure 14). To verify that MRgFUS was able to target the clot, thermometry and acoustic radiation force imaging (ARFI) monitored changes in the clot. During sonication treatment, ARFI measured displacements up to $20 \mu \mathrm{m}$ at the clot location while the remainder of the brain did not show displacements. This confirmed that cavitation was occurring at the desired target point. Thermometry also confirmed that there was minimal heating and occurred only at the target location. 


\section{SickKids}

THE HOSPITAL FOR

SICK CHILDREN

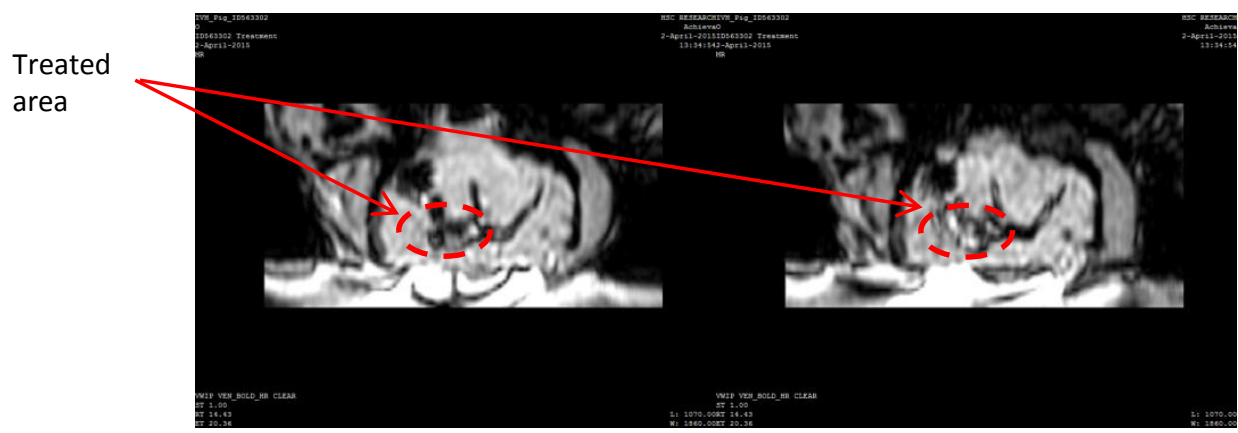

Figure 14 - Left - Pre-treatment, Right - Post-treatment

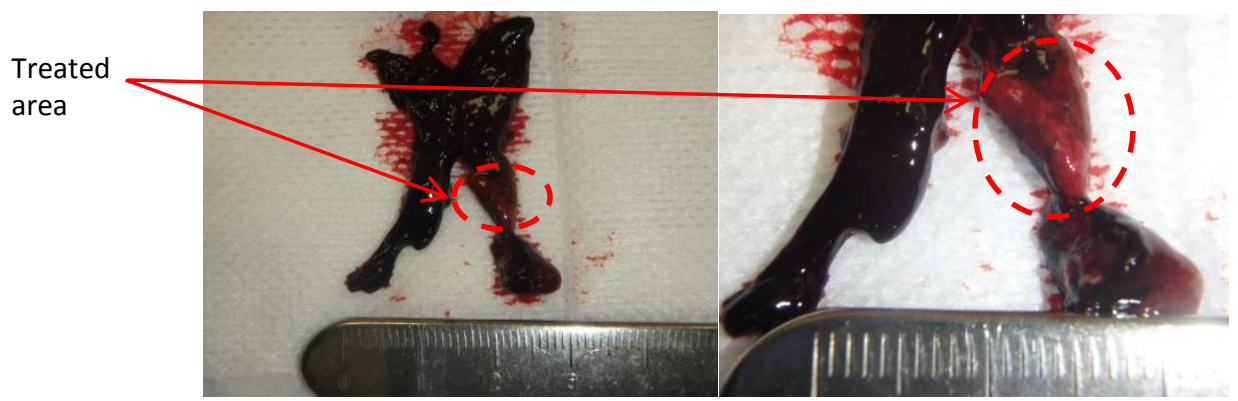

Figure 15 - Treated clot

Based on MR imaging data, the clot images were segmented before and after the treatment. Clot volumes were calculated based 3D volumes derived from the images. Results showed that there was a reduction in clot volume by $34 \%$ and $23 \%$ in the two last IVH piglets (gross volume changes from 5795 $\mathrm{mm}^{3}$ to $3772 \mathrm{~mm}^{3}$ and $3904 \mathrm{~mm}^{3}$ to $3004 \mathrm{~mm}^{3}$ ) (Figure 15). Two earlier treated animals did not have a noticeable clot change where it is hypothesized that this was due to a smaller cranial window that prevented acoustic transmission into the clot.

The piglets were sacrificed and brains were extracted for histological analysis. Gross examination of the brains revealed no damage to the scalp and surface of the brain. The brain samples were perfusion fixed and set in paraffin blocks for H\&E staining. Pathological analysis is pending to determine if there were areas of collateral damage from thrombolysis treatment. 


\section{Conclusions and future work:}

Two cadaveric pediatric skulls have been obtained and characterized. The results confirmed our hypothesis that ultrasound propagation seems easier through these skulls than through adult skulls, especially for the case of very young patients. They also emphasized the need to avoid the sagittal suture for the 8 year-old skull. The method developed in this work is capable of creating maps of acoustic properties on the skull surface, which to our knowledge has not yet being presented in the literature. As clearly indicated by the analysis of the effects of the bone sutures in the 8 year-old skull, having a better understanding of the "regional" acoustic properties can translate to a more effective ultrasound focusing. This observation may have also implications in the use of focused ultrasound in adults. Further studies can be conducting using a similar setup using calvaria of adults.

Two phantom skulls have been manufactured based on the CT dataset of the cadaveric skulls. An 8-year old phantom skull has been characterized and compared to the real specimen. The results suggest the need to refine the 3-layered phantom structure to have a closer match with the real skull. The next step will be to characterize the neonate monolayered phantom skull. After adjustment of the composition of the 8-year old phantom skull, if the results of the 2 phantom skulls are close to the real ones, other phantoms will be manufactured increasing our library of acoustic properties of the skull according to the age of the patient from 2 to 16 years old, with one phantom per year of age.

The speed of sound has been calculated for the 2 skulls and is lower than the adult skull speed of sound. However the speed of sound of the 8-year old skull matches with the prediction model based on the bone density.

The first observations made on the neonate skull results led us to consider not refocusing through this skull when performing non-thermal ultrasound treatments. An IVH piglet model was designed and four animals were initially treated. Pathological analysis is still pending. 
THE HOSPITAL FOR

SICK CHILDREN

\section{Communications:}

Oral presentation:

E. Constanciel Colas, A.C. Waspe, C. Mougenot, T. Looi, S. Pichardo, J.M. Drake, Mapping of insertion losses and time-of-flight delays of pediatric skulls using a clinical MR-guided High Intensity Focused Ultrasound system, $15^{\text {th }}$ International Symposium of Therapeutic Ultrasound (ISTU 2015), Utrecht, April $15^{\text {th }}-18^{\text {th }}, 2015$.

E. Constanciel Colas, A.C. Waspe, C. Mougenot, T. Looi, S. Pichardo, J.M. Drake, Non-invasive treatment of pediatric neurological disorders using Magnetic Resonance-guided Focused Ultrasound: Acoustic characterization of a neonate skull using a clinical HIFU system, 27th International Symposium on Paediatric Surgical Research, Toronto, September $22^{\text {nd }}-23^{\text {rd }}, 2014$.

\section{Poster:}

T. Looi, A.C. Waspe, C. Mougenot, K. Hynynen, J.M. Drake, MRgFUS Thrombolysis of Intraventricular Hemorrhagic (IVH) Clots and Hydrocephalus Treatment in a Porcine Model: Acute and Chronic Results, 15th International Symposium of Therapeutic Ultrasound (ISTU 2015), Utrecht, April 15th-18th,2015.

E. Constanciel Colas, A.C. Waspe, C. Mougenot, T. Looi, S. Pichardo, J.M. Drake, Acoustic characterization of a neonate skull using a clinical MR-Guided High Intensity Focused Ultrasound System for pediatric neurological disorder treatment planning, Annual Brain and Mental Health Day Conference, Toronto, April 23 $3^{\text {rd }}, 2015$.

E. Constanciel Colas, A.C. Waspe, C. Mougenot, T. Looi, S. Pichardo, J.M. Drake, Acoustic characterization of a neonate skull using a clinical MR-Guided High Intensity Focused Ultrasound System for pediatric neurological disorder treatment planning, $4^{\text {th }}$ International Symposium Current and Future Applications of Focused Ultrasound, Washington, October $12^{\text {th }}-16^{\text {th }}, 2014$. 
VII. References:

[1] A. Wydra and R. G. Maev, "A novel composite material specifically developed for ultrasound bone phantoms: cortical, trabecular and skull," Phys. Med. Biol., vol. 58, no. 22, p. N303, Nov. 2013.

[2] B. Zaporzan, A. C. Waspe, T. Looi, C. Mougenot, A. Partanen, and S. Pichardo, "MatMRI and MatHIFU: software toolboxes for real-time monitoring and control of MR-guided HIFU," J. Ther. Ultrasound, vol. 1, no. 1, pp. 1-12, Jun. 2013.

[3] F. J. Fry and J. E. Barger, "Acoustical properties of the human skull," J. Acoust. Soc. Am., vol. 63, no. 5, pp. 1576-1590, May 1978.

[4] S. Pichardo, V. W. Sin, and K. Hynynen, "Multi-frequency characterization of the speed of sound and attenuation coefficient for longitudinal transmission of freshly excised human skulls," Phys. Med. Biol., vol. 56, no. 1, p. 219, Jan. 2011.

[5] C. W. Connor, G. T. Clement, and K. Hynynen, "A unified model for the speed of sound in cranial bone based on genetic algorithm optimization," Phys. Med. Biol., vol. 47, no. 22, pp. 3925-3944, Nov. 2002.

[6] A. M. Loyd, C. Van Ee, M. B. Panzer, B. S. Myers, and C. R. Bass, "Skull Biomechanics," in Orthopaedic Biomechanics, CRC Press, 2012, pp. 121-152.

[7] T. Looi, A. Waspe, C. Mougenot, J. Amaral, M. Temple, K. Hynynen, and J. Drake, "Porcine pilot study of MRI-guided HIFU treatment for neonatal intraventricular hemorrhage (IVH)," in AIP Conference Proceedings, 2012, vol. 1503, pp. 233-238.

[8] T. Looi, A. Waspe, A. James, K. Hynynen, and J. Drake, "Intraventricular hemorrhage clot lysis using MR-guided focused ultrasound in a neonatal piglet model," Proc 2013 AANSCNS Sect. Pediatr. Neurol. Surg., 2013. 